\title{
Theranostic radioimmunotherapy (RIT) combined with immunotherapy: The best way to go?
}

\section{Jean-François Chatal* \\ Groupement d'Intérêt Public Arronax, Nantes, France}

The clinical efficacy of radioimmunotherapy has been clearly documented in non-Hodgkin B-cell lymphoma (NHL) even if, due to a combination of factors, it has not been widely adopted by the oncohematologists community particularly because of the competitive maintenance treatment using rituximab [1]

Some quite promising results have been also obtained in solid tumors despite their relative radioresistance. For treatment of metastatic cancers, single-injection RIT is not realistic for a substantial efficacy. Fractionation of injected activity allows to reduce hematologic toxicity due to a faster bone marrow repair than tumor cell repair. It results that bone marrow can support the injection of higher cumulative activity allowing a higher tumor dose and better efficacy especially for smallsize tumors [2].

RIT with anti-PMSA- ${ }^{177} \mathrm{Lu}-J 591$ antibody has clearly shown the interest of fractionation in treatment of patients with metastatic castration resistant prostate cancer. An increase of $14 \%$ of fractionated cumulative activity with regard to a single injection led to a slight decrease in hematologic toxicity and an impressive overall survival gain from 21.8 to 45.3 mo with respectively low dose and maximal tolerated dose, even if such improvement should be cautiously considered due to the small number of patients [3].

Another promising approach is the use of pretargeting technology. In a clinical study, anti-carcinoembryonic antigen (CEA)/antidiethylenetriamine pentaacetic acid (DTPA) -indium bispecific antibody, followed 4 days later by a ${ }^{131}$ I-labeled bivalent hapten were injected in patients with advanced, progressive medullary thyroid cancer. Forty-seven percent of patients, defined as biologic responders by a more than $100 \%$ increase in calcitonin doubling time, experienced significantly longer survival than nonresponders (median OS, 159 v 109 months) [4]. In a recent preclinical study, a novel three-step pretargeted fractionated radioimmunotherapy (PRIT) strategy based on a glycoprotein A33 (GPA33)-targeting bispecific antibody (bsAb) and a small-molecule radioactive hapten $\left({ }^{177} \mathrm{Lu}\right.$-DOTA-Bn) were injected in nude mice bearing subcutaneous GPA33-positive SW1222 xenografts. Impressively, 100\% complete responses and 100\% cures without any treatment-related toxicities were observed warranting implementation of a clinical study in patients with metastatic colorectal cancer [5].

\section{Need for stratifying patients for RIT}

Unfortunately, due to a great intratumoral phenotypic heterogeneity among patients and among metastases in a same patient, RIT can be unefficient in patients who have a low tumor antigenic expression in metastases resulting in no benefit for them and useless high cost. Immunohistochemistry analysis and quantitative polymerase chain reaction analysis of tumor biopsies can identify patients with the highest chances of efficient RIT but they do not allow whole-body mapping of tumor antigenic expression and thus their capacity to predict efficient treatment remains limited.

Immuno-PET with antibodies labeled with positron-emitting radionuclides such as zirconium-89 or iodine-124, offers a non invasive quantitative imaging for evaluation of tumor antigenic expression in all metastases in a patient and thus can help select patients for an efficient RIT [6]. Immuno-PET, using ${ }^{89} \mathrm{Zr}$-anti-HER2 antibody (trastuzumab) proved to be useful in selecting patients HER2+ in accordance with immunohistochemistry and to predict response to trastuzumab therapy [7].

Large multicentre randomized clinical trials need to be implemented before stating that immuno-PET is really useful for patients stratification before RIT.

\section{Need for combination therapy}

It is well established that ionizing radiation can stimulate the immune system in several ways. As a result, radiotherapy and especially targeted radiotherapy including RIT can contribute to shift an immunosuppressive tumor microenvironment to a more favorable immune stimulatory effect. As a result it makes sense to combine targeted radiotherapy with immunotherapy for a better efficacy.

A therapeutic vaccine, PSA-TRICOM (PROSTVAC) designed to induce activation of $\mathrm{T}$ cells specific against prostate-specific antigen (PSA) has been combined with samarium-153-EDTMP (Quadramet ${ }^{\star}$ ) in a randomized phase 2 trial in patients with metastatic castrationresistant prostate cancer without visceral metastases. Interestingly a significant increase in Progression Free Survival (PFS) was found with evidence of serum PSA decline only in the combination arm [8]. This was the first clinical trial combining a radiopharmaceutical agent with an immunotherapy modality. Another ongoing randomized clinical trial combines Sipuleucel-T, another vaccine designed to induce activation of T cells specific against prostate-specific antigen PAP (Prostatic Acid Phosphatase) with radium-223 (Xofigo ${ }^{\circ}$ ) vs Sipuleucel-T alone and is currently recruiting for a completion date in 2020.

Other clinical trials combining RIT and immunotherapy are planned for the coming years.

In conclusion, for the last 2 or 3 decades a lot of clinical studies performed with different antibodies in hundreds of patients have shown

Correspondence to: Jean-François Chatal, MD, $\mathrm{PhD}$, Groupement d'Intérêt Public Arronax, Nantes, France, E-mail : Jean-Francois.Chatal@univ-nantes.fr

Received: July 20, 2017; Accepted: August 17, 2017; Published: August 21, 2017 
a clinical efficacy in sensitive lymphomas and solid tumors especially in the situation of small-size tumors [9]. In the near future it will be useful to select patients for RIT on the basis of immune-PET imaging using the same antibody labeled with a positron-emitting radionuclide. Finally the efficacy of RIT could be substantially improved when combined to immunotherapy.

\section{References}

1. Chen Q, Ayer T, Nastoupil LJ, Rose AC, Flowers CR (2015) Comparing the costeffectiveness of rituximab maintenance and radio-immunotherapy consolidation versus observation following first-line therapy in follicular lymphoma patients. Value Health 18: 189-197. [Crossref]

2. DeNardo GL, Schlom J, Buchsbaum DJ, Meredith RF, O'Donoghue JA, et al. (2002) Rationales, evidence, and design considerations for fractionated radioimmunotherapy. Cancer 94: 1332-1348. [Crossref]

3. Batra JS, Karir BS, Vallabhajosula S (2015) Fractionated dose radiolabeled antiprostate specific membrane antigen (PSMA) radioimmunotherapy (177Lu-J591) with or without docetaxel for metastatic castration-resistant prostate cancer (mCRPC). $J$ Clin Oncol 33: 194. [Crossref]
4. Chatal JF, Campion L, Kraeber-Bodéré F, Bardet S, Vuillez JP, et al. (2006) Survival improvement in patients with medullary thyroid carcinoma who undergo pretargeted anti-carcinoembryonic-antigen radioimmunotherapy: a collaborative study with the French Endocrine Tumor Group. J Clin Oncol 24: 1705-1711. [Crossref]

5. Cheal SM, Fung EK, Patel MV, Punzalan B, Xu H, et al. (2017) Curative multi-cycle radioimmunotherapy monitored by quantitative SPECT/CT-based theranostics, using bispecific antibody pretargeting strategy in colorectal cancer. $\mathrm{J} \mathrm{Nucl} \mathrm{Med}$. [Crossref]

6. Kraeber-Bodere F, Bailly C, Chérel M, Chatal JF (2016) ImmunoPET to help stratify patients for targeted therapies and to improve drug development. Eur J Nucl Med Mol Imaging 43: 2166-2168. [Crossref]

7. Gebhart G, Lamberts LE, WimanaZ, Garcia C, Emonts P, et al. (2016) Molecular imaging as a tool to investigate heterogeneity of advanced HER2-positive breast cancer and to predict patient outcome under trastuzumab emtansine (T-DM1) : the ZEPHIR trial. Ann Oncol 27: 619-624. [Crossref]

8. Heery CR, Madan RA, Stein MN, Stadler WM, Di Paola RS, et al. (2016) Samarium153-EDTMP (Quadramet $\AA$ ) with or without vaccine in metastatic castration-resistant prostate cancer:a randomized phase 2 trial. Oncotarget 7: 69014-69023. [Crossref]

9. Kraeber-Bodéré F, Barbet J, Chatal JF (2016) Radioimmunotherapy: From Current Clinical Success to Future Industrial Breakthrough? J Nucl Med 57: 329-331. [Crossref]

Copyright: $\odot 2017$ Chatal JF. This is an open-access article distributed under the terms of the Creative Commons Attribution License, which permits unrestricted use, distribution, and reproduction in any medium, provided the original author and source are credited. 